\title{
Cáncer de laringe y Virus del papiloma humano en adultos del Hospital Escuela Universitario, Honduras
}

\author{
Carmen Rosario Estrada Núñez ${ }^{1}$ \\ Juan Carlos Barrientos Augustinus ${ }^{2}$ \\ Annabelle Ferrera Boza ${ }^{3}$ \\ Edna Maradiaga ${ }^{4}$
}

\section{RESUMEN}

El cáncer de laringe es el más frecuente en cabeza y cuello; recientemente se plantea la infección por virus del papiloma humano (VPH) en la carcinogénesis. El objetivo del estudio fue establecer la presencia del VPH en pacientes con carcinoma de laringe en el Hospital Escuela Universitario (HEU) desde marzo de 2012 a marzo de 2015.

Se hizo un estudio retrospectivo. Previa autorización del Departamento de Patología se revisaron los diagnósticos histopatológicos de pacientes con carcinoma de laringe en HEU. El universo fue de 91 pacientes con carcinoma de laringe; la muestra fue de 30 pacientes.

Se identificó el ADN del VPH mediante la reacción en cadena de la polimerasa (PCR) y genotipificación molecular utilizando sondas marcadas mediante hibridación reversa LIPA (INNO-LIPA HPV Genotyping Extra). Se realizó tabulación y análisis en EPIINFO 3.5.4; se obtuvo frecuencias, porcentajes, medidas de tendencia central y de significancia estadística.

Los resultados indican distribución por género, masculino 26/30 (86.6 \%) y femenino $4 / 30$ (13.3 \%); edad media para VPH negativos (62.7 años) y para VPH positivos (54.8 años). Procedentes del área rural 19/30(63.3\%). Cofactores predominantes: el tabaquismo en 22/30 (73.3\%) y alcoholismo 20/30 (66.6 \%). El sitio anatómico

\footnotetext{
${ }_{1}^{1}$ Beneficiaria de una beca de estudiante de posgrado de la DICYP. Residente del 4to año del Posgrado de Otorrinolaringología (ORL) de la Facultad de Ciencias Médicas (FCM) UNAH: crestrada7@yahoo.com

${ }^{2}$ Asesor. Profesor del Posgrado de ORL de la FCM-UNAH,

${ }^{3}$ Asesora. Profesora de la Escuela de Microbiología UNAH,

${ }^{4}$ Asesora. Profesora de la Unidad de Investigación Científica FCM-UNAH: edjamar3006@yahoo.com
} 
más afectado fue la glotis 16/30(53.3 \%). Histológicamente predominó el carcinoma epidermoide moderadamente diferenciado 21/30 (70 \%). Al momento del diagnóstico12/30(40\%) se encontraban en estadio III.

El porcentaje de VPH fue del $43.3 \%$ (13/30), IC 95\%(25.5-62.6), $p<0.001$. Se identificaron genotipos de alto riesgo $(16,31,33,51,52,53,56,58)$ y bajo riesgo $(6,11,71$ y 74), predominando VPH 11 (23\%) no oncogénico, seguido del VPH 16 (15.4\%) oncogénico. Hubo coinfección de dos o más genotipos.

La conclusión es que el porcentaje de VPH fue del $43.3 \%$ con predominio de VPH 11 y 16. Debido a la muestra reducida y a la variabilidad de los resultados en estudios internacionales, se recomiendan estudios futuros con casuística más grande.

Palabras clave: neoplasias laríngeas, papilomavirus humano, PCR.

\section{ABSTRACT}

Introduction: laryngeal cancer is the most frequent in head and neck, recently HPV is involved in its carcinogenesis. Objective: To identify the presence of human papillomavirus (HPV) in patients with larynx carcinoma at HEU from March 2012 until March 2015.Methods: A retrospective study was performed. Under authorization of the Pathology Department, the histopathological studies from patients already diagnosed having laryngeal carcinoma during the period from March 2012- March 2015 were reviewed. A universe of 91 patients with larynx carcinoma was obtained. In the selected 30 patients whose specimens were preserved in paraffin blocks, HPV DNA identification by polymerase chain reaction (PCR) followed by molecular genotyping using hybridization probes by reverse LIPA (INMO - LIPA HPV Genotyping Extra) were accomplished.

Results: Gender distribution: 26/30 (86.6 \%) men and 4/30 (13.3\%) women with a mean age of 62.7 yearsin HVP positive patients was 54.8 years .Coming largely from the rural areas19/30(63.3\%). The risk co-factors identified weresmoking22/30(73.3 $\%$ ) and alcoholism 20/30(66.6 \%). The most affected anatomic site: the glottis 16/30 (53.3 \%). Histologically prevailed in 21/30 (70 \%) of patients moderately differentiated squamous cell carcinoma. Most patients were in stage III12/30(40\%) at the diagnosis time, $(p=0.09)$.HPV prevalence was 13/30(43.3\%), IC95\% (25.5-62.6), $p<0.001$. High 
risk genotypes $(16,31,33,51,52,53,56,58)$, as well as low risk $(6,11,71$ and 74$)$ were identified. Non-oncogenic HPV $11(23 \%)$ was the most frequent type encountered and the oncogenic HPV 16 (15.4\%). Co-infection was noted with two or more genotypes.

Conclusions: Prevalence HPV was 43.3\%. HPV 11 and 16 were the most frequent. Due to a reduce sample and results variability among international studies, it is not possible to establish a clear association between HPV and laryngeal cancer. Further studies with more representative sample are required and recommended.

Keywords: Laryngeal neoplasm, Human Papillomavirus, PCR. 


\section{INTRODUCCIÓN}

El carcinoma de células escamosas de cabeza y cuello constituye la sexta causa más frecuente de malignidad en esta área alrededor del mundo; de estos, el carcinoma de laringe es el segundo en orden de aparición, constituyendo el $4.5 \%$ de la totalidad de los carcinomas y $28 \%$ de las tumoraciones del tracto aero-digestivo superior, solo superado por el carcinoma de cavidad de oral. (Lingbin et al., 2015) A nivel mundial se calcula que en 2012 hubo 156,877 mil casos nuevos de cáncer de laringe y 83,736 mil muertes. Con una incidencia de 2.1/100,000 y una mortalidad de 1.1/100,000 (Lingbin et al., 2015). La laringe puede albergar diferentes histologías de tumores malignos primarios (adenocarcinomas, sarcomas, linfomas tumores carcinoides), pero el más común es el carcinoma epidermoide ( $90 \%)$; de hecho, la laringe ocupa el segundo lugar de sitio primario en la región de cabeza y cuello para el desarrollo de carcinomas epiteliales. (Xiangwei et al., 2013).

El cáncer de laringe tiene alta mortalidad en etapas avanzadas, pero es potencialmente curable si se diagnostica en estadios tempranos y es tratado adecuadamente (Suarez et al., 2009). Los factores de riesgo clásicos para el desarrollo de cáncer de laringe son el tabaco y el alcohol, pero se han vinculado otros factores como la infección del virus del papiloma humano (VPH), el reflujo gastroesofágico y los vinculados con exposición ocupacional a polvo de madera y asbesto. (Hernández, Castillo, Gómez, Rodríguez, Fernández, 2014).

La relación del VPH con la aparición de cáncer de cabeza y cuello ha sido firmemente establecida en las últimas décadas. Los casos confirmados histológicamente con cáncer epidermoide y positivos por VPH están asociados a personas jóvenes, sexo masculino y comportamiento sexual de alto riesgo, como múltiples parejas sexuales (más de 6) y el sexo oral. (Quintero et al., 2013).

EL VPH es un virus compuesto por una pequeña cadena circular doble de ácido desoxirribonucleico (ADN) que forma parte de una gran familia de papiloma virus (Papillomaviridae) que infectan vertebrados, desde aves hasta seres humanos, y ocasionan neoplasias epiteliales malignas y benignas; reciben su nombre de acuerdo a sus huéspedes naturales, por ejemplo VPH bovino, murino y humano. Es específico de cada especie que infecta y esta especificidad es absoluta. (Fuente-Villarea, Guzmán-López, Barboza-Quintera, Gonzalez-Ramirez, 2010).

En función de estas características, los diversos tipos de VPH se numeran para 
distinguirlos; hasta el momento se han identificado más de 200 tipos distintos de VPH. (Fuente-Villarea, 2010). Pero, hay tres grupos principales asociados con el desarrollo de lesiones mucosas en el tubo digestivo, las vías aéreas y el aparato genital. Los VPH 6 y 11 son responsables de la papilomatosis laríngea recurrente y forman parte de una clase considerada con bajo potencial maligno en comparación con otros grupos, como el que incluye los tipos 16, 31, 3 y 18, considerados por la Organización Mundial de la Salud (OMS) y la International Agency For Research on Cancer (IARC). (Liu et al., 2013).

Se estima que las infecciones por virus juegan un rol en al menos $11 \%$ de casos nuevos de cáncer alrededor del mundo, la mayoría (>85\%), ocurren en países en vías de desarrollo, con pobres condiciones sanitarias, falta de acceso a vacunas, coexistencia de otros factores de riesgo $(\mathrm{VIH}$-sida) y programas deficientes de detección temprana de cáncer. En países desarrollados, este porcentaje se reduce a un $4 \%$.

El curso lento de la inducción cancerígena, típicamente en varios años después de la infección viral, sugiere que esta por sí sola no es capaz de la transformación maligna, es necesario, como se mencionó antes, la coexistencia de otros factores de riesgo. Así, el objetivo de esta investigación fue establecer la asociación entre la infección del VPH y el desarrollo de cáncer de laringe en pacientes del HEU. El presente estudio constituye el primero que se realiza en Honduras, solo se registra un estudio de genotipificación del VPH en pacientes con papilomatosis laríngea realizado en el servicio de otorrinolaringología (ORL) del HEU en año 2008 (Bonilla-Ramírez, Barrientos-Augustinus, Ferrera-Boza 2008). Por tanto, se considera que este proyecto de investigación es una oportunidad valiosa para corroborar los datos ya establecidos internacionalmente, ya que permitirá obtener datos nacionales que guiarán en la toma de medidas preventivas y terapéuticas eficaces y oportunas acorde a la realidad de nuestro entorno.

\section{METODOLOGÍA}

\section{Tipo de estudio, población estudiada y recolección de especímenes}

Se trató de un estudio retrospectivo, el cual fue llevado a cabo en la consulta externa y sala de hospitalización del servicio de ORL del HEU durante un periodo de 4 años (marzo de 2012-marzo de 2015). Se hizo revisión de los libros de registro del Departamento de Patología previa autorización de su jefatura. Se obtuvo un total de 91 
pacientes con diagnóstico histológico de carcinoma de laringe. Se seleccionó una muestra de 30 pacientes a conveniencia por costos elevados y de forma proporcional al número de casos por año; luego se obtuvieron los datos sociodemográficos, factores de riesgo, diagnóstico histológico, sitio anatómico afectado y estadio de los expedientes clínicos, reportes de patología y tomografías computarizadas, para lo cual se utilizó un formulario previamente validado con el que se realizó el vaciado de la información.

El procesamiento de las muestras se realizó mediante el método molecular de amplificación del DNA del virus por PCR seguido de la genotipificación por hibridación reversa (INNO-LIPA HPV Genotyping Extra).

En el presente trabajo, previamente a la amplificación, se llevó a cabo la extracción de ADN de biopsias de laringe incluidas en bloques de parafina y unas pocas en formalina. De cada bloque se utilizaron 3 cortes o secciones de $5 \mu \mathrm{m}$ de grosor, a los cuales se les retiró la mayor cantidad posible de parafina con la ayuda de un bisturí y se colocó el material dentro de un tubo Eppendorf de 1,5 ml. La extracción de ADN se realizó con el QIAamp DNA mini kit (QIAGEN, Alemania), siguiendo las indicaciones del fabricante.

La calidad de los ADN extraídos fue probada mediante PCR para la detección del gen humano HLA-DPB1. Una vez extraído el ADN, se procedió a realizar la detección y tipificación de VPH. Para ello se utilizó el Kit INNO.LiPA Genotyping Extra, que está basado en el principio de hibridación reversa. Parte de la región L1 del genoma del HPV es amplificada utilizando los primeros SPF10 y los amplicones biotilinados resultantes se desnaturalizan e hibridizaron con sondas de oligonucleótidos específicas. Un juego adicional de cebadores se utiliza para amplificar el gen humano HLA-DPB1 para monitorear la calidad de la muestra y la extracción. Todas las sondas se inmovilizaron como líneas paralelas en membranas de nitrocelulosa.

Los productos de amplificación fueron posteriormente hibridizados utilizando una única tira de nitrocelulosa, en donde 28 sondas de DNA secuencia específica de tipos de HPV y 4 controles están inmovilizados. Después de la hibridación y un lavado astringente, el conjugado estreptavidina-fosfatasa alcalina es adicionado; este se une a los híbridos biotinilados formados previamente. La incubación con cromógeno BCIP/NBT da lugar a un precipitado púrpura, siendo el resultado interpretado de manera visual.

El ensayo identifica cualitativamente los tipos de VPH clasificados de alto riesgo 
oncogénico $16,18,26,31,33,35,39,45,51,52,53,56,58,59,66,68,73$ y 82; así como la detección de los tipos de VPH de bajo riesgo más comunes $6,11,40,43,44$, $54,69,70,71$ y 74 . Para el análisis de los datos se utilizaron medidas de tendencia central, frecuencias, porcentajes, tablas, Odds Ratio; se realizó cruce y análisis de más de dos variables. La información de los datos obtenidos fue manejada con la confidencialidad debida y respeto a los pacientes.

\section{RESULTADOS}

Se incluyeron en este estudio un total de 30 pacientes con carcinoma de laringe. Con respecto a las características sociodemográficas, el sexo masculino fue el más afectado. La edad media de los pacientes con cáncer de laringe fue de 62.7 años y 54.8 años para los pacientes VPH positivos $(p=0.09)$, ligeramente menor a la esperada en los pacientes con los factores de riesgo clásicos (tabaquismo y alcoholismo), la mayoría provenía del área rural 19/30(63.3\%) (Ver cuadro 1).

Cuadro 1. Distribución de las características sociodemográficas de los pacientes con carcinoma de laringe en el Servicio de ORL del HEU. Marzo 2012-Marzo 2015.

\begin{tabular}{|lcl|}
\hline Característica & Frecuencia(n:30) & Porcentaje (\%) \\
\hline Género & & \\
\hline Masculino & 26 & 86.6 \\
Femenino & 4 & 13.3 \\
\hline Grupo Etario & & \\
\hline $\mathbf{2 5 - 3 4}$ & 2 & 6.6 \\
$\mathbf{3 5 - 4 4}$ & 2 & 6.620 \\
$\mathbf{4 5 - 5 4}$ & 6 & 20 \\
$\mathbf{5 5 - 6 4}$ & 6 & 46.6 \\
$\mathbf{6 5}$ y más & 14 & \\
Promedio & $62.7+/-3$ & \\
Rango & $31-84$ & \\
\hline Procedencia & & 63.3 \\
\hline Rural & 19 & 36.6 \\
Urbana & 11 & \\
\hline Ocupación & & 83.3 \\
\hline Agricultura & 25 & 3.3 \\
Ingeniería & 1 & 10 \\
Ama de casa & 3 & 3.3 \\
Sastrería & 1 & \\
\hline
\end{tabular}


Según el American Joint Committee (AJC) y la Unión Internacional Contra el Cáncer (UICC), la laringe se divide en tres regiones: supra glotis, glotis y sub glotis. En el presente estudio el sitio anatómico más afectado fue la glotis 16/30(53.3 \%) (Ver figura 1).

Figura 1. Distribución de acuerdo a sitio anatómico afectado. Pacientes con carcinoma de laringe.

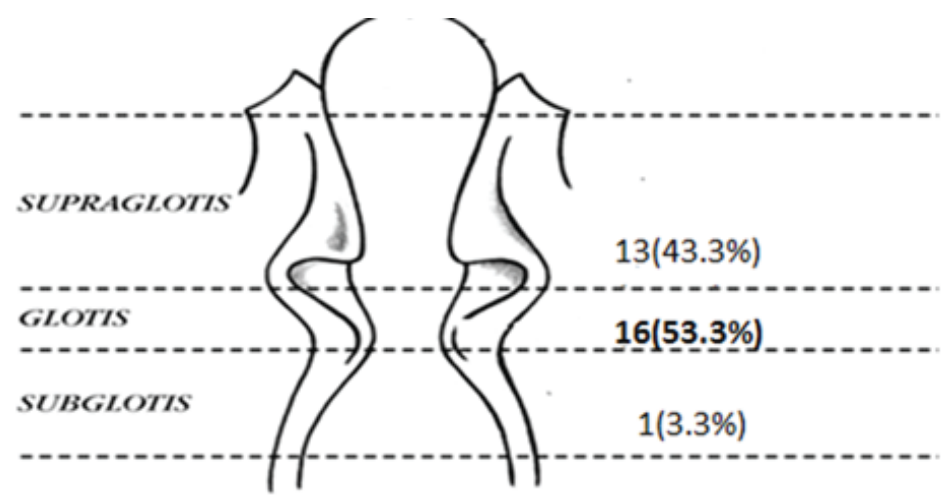

Cantar y Hablar. Conocimientos Generales de la voz. Editorial Paidotribo. España 2005.

Con respecto al tipo histológico se encontró que el carcinoma epidermoide moderadamente diferenciado invasor fue el predominante en $21 / 30(70 \%)$ de los casos y solo $3 / 30(23 \%)$ correspondían a carcinomas bien diferenciados queratinizantes. En estos últimos se encontró curiosamente VPH de bajo riesgo (11 y 6) y al momento del diagnóstico se encontraban en estadios clínicos tempranos (I y II), en comparación con los afectados por VPH de alto riesgo que se encontraban en estadios avanzados de la enfermedad (III y IV), p=0.09 (ver cuadro 2).

Cuadro 2. Distribución de acuerdo al estadio clínico al momento del diagnóstico .de pacientes con carcinoma de laringe. Servicio de ORL-HEU. Marzo 2012-marzo 2015. N: 30.

\begin{tabular}{|l|c|c|}
\hline Estadio Clínico & Frecuencia_(n:13) & Porcentaje (\%) \\
\hline I & 3 & 10 \\
\hline II & 6 & 20 \\
\hline III & 12 & 40 \\
\hline IV & 9 & 30 \\
\hline
\end{tabular}


En este estudio hay factores de riesgo asociados: tabaquismo, alcoholismo, exposición a sustancias tóxicas y predisposición genética; se desestimó el factor conducta sexual riesgosa, ya que solo en dos de los pacientes positivos por el virus se encontró reporte de este dato en particular, ambos por múltiples parejas sexuales y sexo oral. En el resto no estaba consignado en los expedientes clínicos o los pacientes y familiares se negaron a revelar esta información. Lo anterior es sugestivo del sinergismo en la carcinogénesis que se cumple en estos pacientes al coexistir dos o más factores de riesgo (ver cuadro 3 ).

Cuadro 3. Distribución de los factores de riesgo en los pacientes con cáncer de laringe y VPH positivos. Servicio de ORL-HEU. Marzo 2012-Marzo 2015.

\begin{tabular}{|l|l|l|l|l|}
\hline Factores de Riesgo & VPH positivo N (\%) & VPH negativo N (\%) & OR & IC (95\%) \\
\hline $\begin{array}{l}\text { Tabaquismo } \\
\mathrm{Si} \\
\mathrm{No}\end{array}$ & $\begin{array}{c}10(45.5) \\
3(37.5)\end{array}$ & $\begin{array}{c}12(54.5) \\
5(62.5)\end{array}$ & 1.38 & $0.26-7.2$ \\
\hline Alcoholismo & $8(40)$ & $12(60)$ & & \\
\hline $\mathrm{Si}$ & $5(50)$ & $5(60)$ & 0.67 & $0.14-3.07$ \\
$\mathrm{No}$ & & & & \\
\hline $\begin{array}{l}\text { APF de cáncer de } \\
\text { laringe }\end{array}$ & $3(75) 10(38.5)$ & $\begin{array}{c}1(25) \\
16(61.5)\end{array}$ & 4.8 & $0.4-52.7$ \\
$\mathrm{Si}$ & & & & \\
$\mathrm{No}$ & $3(75)$ & $1(75)$ & 4.8 & $0.4-52.7$ \\
\hline $\begin{array}{l}\text { Sustancias Tóxicas } \\
\mathrm{Si} \\
\text { No }\end{array}$ & $10(38.5)$ & $16(61.5)$ & & \\
\hline
\end{tabular}

De los 30 pacientes incluidos en este estudio, 13/30 (43.3\%) fueron positivos por ADN de VPH, IC 95\% (25.5-62.6), p<0.001. Fue utilizado el método de LIPA (Genotyping kit HPV GP Versión 2) para el procesamiento de las muestras. Se muestra la detección molecular del VPH por electroforesis (ver figura 2). Los genotipos de VPH encontrados fueron: 6, 11, 71 y 74 (VPH de bajo riesgo); 16, 31, 33, 51, 52, 53, 56 y 58 (VPH de alto riesgo). De estos, VPH 11 (23\%) fue el predominante. 
Figura 2. Electroforesis en la detección molecular de VPH en carcinoma de laringe. Pacientes servicio de ORL de HEU. Marzo 2012-marzo 2015.

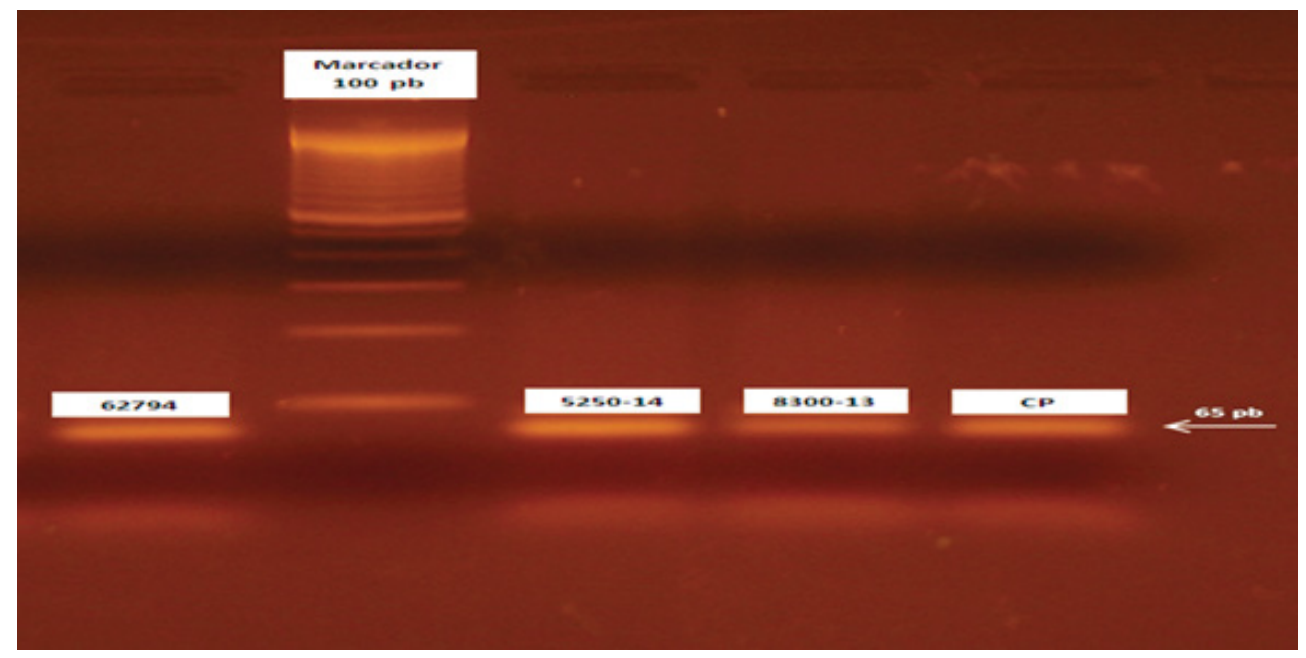

\section{DISCUSIÓN}

En este estudio, al igual que en la literatura internacional, se pudo observar una probable asociación entre el VPH y el carcinoma de laringe, pues fue encontrada una prevalencia de $43.3 \%$ ( $p=0.001)$; más alta incluso que la encontrada en estudios realizados en Colombia (24\%) (Hernández, 2014). China (30.7 \%) (Fuente-Villarea, 2010) y Chile (25\%) (Suarez, 2009).

La variabilidad en los resultados sigue estando presente, ya que se ha reportado una positividad que oscila entre el $13 \%$ y $60 \%$ (Suarez, 2009, Torrente, 2011, Chitose et al., 2010). En una revisión sistemática publicada en Colombia en el año 2013, se incluyeron 60 estudios; 5,046 muestras de pacientes con cáncer de cabeza y cuello (orofaringe, cavidad oral y laringe), en los que se utilizó el método de PCR para la detección del ADN del VPH, lo cual mostró una prevalencia aproximada del $25.9 \%$, orofaringe (35.6 \%), cavidad oral (23.5\%) y laringe (24\%). En el año 2009 , The International Agency for Research on Cancer evaluó la información disponible acerca de la carcinogénesis de VPH en cabeza y cuello, concluyendo que hay suficiente evidencia del potencial oncogénico de VPH 16 en cavidad oral, orofaringe y amígdalas, pero aún hay un conocimiento limitado en la laringe (Hernández, 2014), es por eso que es necesario realizar estudios futuros en donde las muestras sean más amplias y, por ende, los resultados con mayor significancia estadística. 
Los genotipos de VPH encontrados fueron los siguientes: VPH de alto riesgo (16, 31, $33,51,52,53,56,58)$ y VPH de bajo riesgo $(6,11,71$ y 74$)$, siendo el VPH 11 (bajo riesgo) el más frecuente en $23 \%$ de los casos; lo cual difiere de la literatura internacional, en donde el VPH 11 está relacionado con la papilomatosis respiratoria recurrente juvenil, neoplasia benigna de laringe con poco o nulo porcentaje de carcinogénesis (0-22\%). En cambio, VPH 16 y 18 son los más frecuentemente identificados como oncogénicos en cáncer de cabeza y cuello (Nelkek, Lysenko, Leszczyszyn, Gerber.2013). En varios de los casos estudiados se observó la coexistencia de dos 0 más genotipos. Fueron reportados $2(15.3 \%)$ casos en los que el genotipo no correspondió a ninguno de los genotipos de VPH identificados por la prueba molecular de LIPA.

Se encontró que los pacientes estudiados presentaban antecedentes de otros factores de riesgo asociados como el tabaquismo en 22/30 (73.3\%) / OR 1.38 IC $95 \%$ (0.26- 7.2) y 20/30 (66.6 \%) / OR 0.67 IC $95 \%$ (0.14- 3.07) tenían antecedentes de etilismo crónico. Con respecto al alcoholismo no encontramos una significancia estadística tan importante, lo cual coincide con la literatura internacional en la cual se asocia la positividad de VPH en pacientes jóvenes con poca tendencia al consumo del alcohol. Y con respecto al tabaquismo, de acuerdo al resultado de la prueba estadística aplicada OR, este puede considerarse como factor de riesgo con mayor significancia estadística, lo cual puede sugerir que este factor de riesgo en particular, junto con la exposición a sustancias tóxicas y la predisposición genética, actúan de forma sinérgica y llevan en el trascurso de los años a la transformación maligna. (Gron, Schultz, Abildgaard ,2011).

Se desestimó el factor "conducta sexual riesgosa", ya que este dato en particular solo estuvo consignado en dos (2) de los expedientes clínicos y los pacientes o sus familiares se negaron a revelar esta información. En diferentes estudios reportan este dato como muy influyente en la infección por el VPH, sobre todo la práctica del sexo oral y la tenencia de múltiples parejas sexuales; (Omland et al., 2014), con respecto al antecedente de papilomatosis respiratoria recurrente, solamente en una (1) de las pacientes (femenina) existía el antecedente dudoso de esta afección, incluso con traqueotomía previa 20 años antes a la revisión del caso. Como fue mencionado antes, existe evidencia científica en la cual se considera a la papilomatosis laríngea recurrente como factor de riesgo para la carcinogénesis. (Rettig E, Kiess AP, Fakhry, 2015).

En este estudio la mayoría de los pacientes fueron diagnosticados en estadios avanzados de la enfermedad, lo cual es similar a lo reportado en la literatura internacional 
(Cheremisina, Choinzonov, Pankova, Mukahamedov, 2015), 9 (30 \%) en estadio IV; $12(40 \%)$ en estadio III; $6(20 \%)$ en estadio II y $3(10 \%)$ en estadio I ( $P=0.09)$. Los pacientes en estadios tempranos presentaban positividad por VPH de bajo riesgo ( 6 y 11).

Con respecto al diagnóstico histológico, la gran mayoría fueron carcinomas epidermoides moderadamente diferenciados invasores (Karatzanis, Psychogios, Woldfahrer, Kapsreiker, Zenk, 2014) y solo dos (2) casos fueron reportados como bien diferenciados queratinizantes, en general de mejor pronóstico; ambos tenían en común positividad por VPH de bajo riesgo (6 y 11) y alto riesgo (52) en uno de los casos.

El sitio anatómico más afectado fue la glotis, con 16/30 (53.3 \%) de los casos, seguido de la supra glotis con 13/30 (43.3\%) de los casos. (Liang, Bin, Hao, Shu, Xueguan, 2014). La mayoría de los pacientes estudiados, $26 / 30$ (86.6\%) fueron del sexo masculino y solo $4 / 30$ (13.3\%) fueron del sexo femenino, lo anterior también es similar a lo encontrado en estudios internacionales en donde el sexo masculino es el más afectado en más del $80 \%$.

La edad media identificada para los 30 pacientes estudiados fue de 62.7 años (rango 31-84), lo cual puede sugerir que los pacientes VPH positivos tienden a ser más jóvenes que los VPH negativos (62.7 vs 54.8 años), $p=0.09$ ligeramente menor a la esperada para la aparición de carcinoma de laringe con los ya conocidos factores de riesgo clásicos (alcoholismo, tabaquismo, etc.).

\section{CONCLUSIONES}

La prevalencia de VPH del $43.3 \%$ encontrada en este estudio, puede sugerir una asociación entre la infección del VPH y la aparición del cáncer de laringe $(P<0.001)$, esta afirmación aún no está claramente establecida, pues existe mucha variabilidad en los resultados internacionales; en este sentido, es importante seguir investigando este tema en particular, ampliar la casuística puede aumentar la significancia estadística. Además, se halló que la infección viral por sí sola no es capaz de inducir malignidad, sino que generalmente están presentes otros factores de riesgo que actúan de forma sinérgica para la carcinogénesis. Una positividad por VPH de más del $40 \%$ invita a continuar investigando de este tema en particular con muestras más amplias, ya que la limitante más importante fue el factor monetario, por lo oneroso que resulta 
el procesamiento de las muestras, lo que permitirá en el futuro proponer medidas preventivas en contra de la infección por VPH e, incluso, plantear la hipótesis de la posible utilidad de la inmunización mediante la vacuna en contra de este virus, lo cual incluiría a hombres y mujeres.

Todo lo anterior tiene el objetivo de dar un manejo oportuno y eficaz a estos pacientes que llegan en etapas avanzadas de la enfermedad, lo cual limita en gran medida la calidad de vida de los mismos, debido a las funciones vitales (respiración, voz, deglución) del órgano principal afectado: la laringe

\section{AGRADECIMIENTO}

A la doctora Vilma Díaz Bonilla, especialista en ORL, por al apoyo brindado en la obtención de la beca de estudiante de posgrado; igualmente, a los pacientes, pues sin ellos no sería posible este estudio.

\section{BIBLIOGRAFÍA}

Bonilla-Ramírez M, Barrientos-Augustinus J, Ferrera-Boza A. Caracterización Clínico-Epidemiológica e Identificación Molecular del Virus del Papiloma Humano en Papilomatosis Respiratoria Recurrente. Revista médica de los Postgrados de Medicina UNAH. 2008; 11(1).

Cheremisina OV, Choinzonov EL, Pankova OV, Mukahamedov MR. Papilomatosis as criteria for the formation of the group al risk of laryngeal cancer. VestnOtorinolaringol. 2015; 80(1): 39-43

Chitose S, Sakazaki T, Ono T, Kurita T, Mihashi H, Nakashima T. Inmunological responses against human papilloma virus and human papilloma virus induced laryngeal cancer. J Laryngol Otol. 2010; 124(6): 659-62

Fuente-Villarea D, Guzman-López S, Barboza-Quintera O, Gonzalez-Ramirez R. Biología del Virus del Papiloma Humano y Técnicas de Diagnóstico. Medicina Universitaria. 2010; 12(49): 231-238.

Gron AL, Schultz JH, Abildgaard J. Malignant degeneration in laryngeal papilomatosis. UgeskrLaeger. 2011; 173: 506-7

Hernández 0, Castillo Esther, Gómez Isabel, Rodríguez José, Fernández Roberto. 
Factores de Riesgo del Cáncer Laríngeo. Rev Ciencias Médicas. 2014; 18(6). Karatzanis A, Psychogios G, Woldfahrer F, Kapsreiker M, Zenk J. Management of locally advanced laryngeal cancer. J Otolaryngol Head Neck. 2014; 43(1): 4 Liang Han, Bin Jiang, Hao Wu, Shu Zhang, Xueguan Lu. Expression and prognostic value of MAGE-A9 in laryngeal squamous cell carcinoma. Int J. ClinExpPathol. 2014; 7(10): 6734-42

Lingbin D, Huizhang L, Chen Z, Rangshou Z, Siwei Z, Wanging C. Incidence and mortality of laryngeal cancer in China, 2011. Chin F Cancer Res. 2015; 27(1): 52-58

Liu H, Li J, Diao M, Cai Z, Yang J, Zeng Y. Statistical analysis of human papillomavirus in a subset of upper aerodigestive tract tumors. J. Med Virol. 2013; 85(10): 1775-85

Nelkek H, Lysenko L, Leszczyszyn J, Gerber H. Human Papilloma Virus and its influencen on head and neck cancer predisposition. PostepyHig Med Dosw. 2013; 15(67): 610-6

Omland T., LieKa, Akre H., Sandlie L.E., Jebsen P., et al. Recurrent Respiratory PapiIlomatosis: HPV Genotypes and Risk OF high-Grade Laryngeal Neoplasia. PLOS ONE. 2014; 9(6): 981-99

Quintero K, Giraldo G, Uribe M, Baena A, López C, Álvarez E, Sánchez G. Human Papilloma Virus types in cases of Squamouscell carcinoma of head and Neck in Colombia. BJ ORL. 2013; 79 (3): 375-81

Rettig E, Kiess AP, Fakhry G. The role of sexual behavior in head and neck cancer: Implications for prevention and therapy. Expert Rev Anticancer Ther. 2015; 15(1): $35-49$

Suarez C, Gil Carcedo L. M., Marco J, Medina J.E., Ortega P, Trinidad J. Tratado de Otorrinolaringología y Cirugía de Cabeza y Cuello. Volumen III. 2a ed. España. Editorial Panamericana ; 2009.

Suarez C, Gil Carcedo L.M., Marco J, Medina J. E., Ortega P, Trinidad J. Tratado de Otorrinolaringología de Cirugía de Cabeza y Cuello. Volumen IV. 2a ed. España. Editorial Panamericana; 2009

Torrente. C., Rodrigo J. P., Haigentz M., Dikkers F.G., Ferlito A. Human Papilloma virus infections in laryngeal cancer. Head Neck. 2011; 33 (4): 581-6

Xiangwei L, Lei G, Huijun L, Jing G, Yu Y, Feng Z, Cong G, Mufei L, Qi J. Human Papillomavirus Infection and Laryngeal Cancer Risk: A Systematic Review and Meta-Analysis. JID. 2013; 479-88 